\title{
Antithyroid Drug-induced Agranulocytosis Complicated by Pneumococcal Sepsis and Upper Airway Obstruction
}

\author{
Naoto Ishimaru ${ }^{1}$, Hisashi Ohnishi ${ }^{1}$, Teruaki Nishiuma ${ }^{2}$, Ryota Doukuni ${ }^{1}$, Kanoko Umezawa ${ }^{1}$, \\ Sachiko Oozone ${ }^{1}$, Emi Kuramoto ${ }^{1}$, Sho Yoshimura ${ }^{1}$ and Saori Kinami ${ }^{1}$
}

\begin{abstract}
Streptococcus pneumoniae is a rare pathogen of sepsis in patients with antithyroid drug-induced agranulocytosis. We herein describe a case of antithyroid drug-induced agranulocytosis complicated by pneumococcal sepsis and upper airway obstruction. A 27-year-old woman who was previously prescribed methimazole for nine months presented with a four-day history of a sore throat. She nearly choked and was diagnosed with febrile agranulocytosis. She was successfully treated with intubation, intravenous antibiotics and granulocyte colony-stimulating factor. Her blood cultures yielded S. pneumoniae. Emergency airway management, treatment of sepsis and the administration of granulocyte colony-stimulating factor can improve the clinical course of antithyroid drug-induced pneumococcal sepsis in patients with airway obstruction.
\end{abstract}

Key words: antithyroid agents, agranulocytosis, pneumococcal infection, airway obstruction, granulocyte colony-stimulating factor

(Intern Med 52: 2355-2359, 2013)

(DOI: 10.2169/internalmedicine.52.0220)

\section{Introduction}

Antithyroid drug (ATD)-induced agranulocytosis (an absolute neutrophil count of $<500 \times 10^{6} / \mathrm{L}$ ) is rare, developing in only $0.1-1.0 \%$ of patients consuming antithyroid drugs (1). However, life-threatening infections reportedly develop in $0.23 \%$ of patients with hyperthyroidism treated with antithyroid drugs (2), with a high mortality rate of $5-14 \%(2,3)$. The development of bacteremia is a known predictor of a poor prognosis in patients with drug-induced agranulocytosis (4).

Currently, Gram-negative bacilli, including Pseudomonas aeruginosa and Klebsiella pneumoniae, are the most common pathogens involved in such infections, followed by Escherichia coli and Staphylococcus aureus (2). The occurrence of pneumococcal bacteremia in neutropenic cancer (5) and HIV-infected (6) patients is well known; however, no previous reports have described patients with ATD-induced agranulocytosis complicated by pneumococcal sepsis. We herein report the successful treatment of a patient with ATD- induced agranulocytosis complicated by pneumococcal sepsis and upper airway obstruction.

\section{Case Report}

A 27-year-old woman presenting with a four-day history of arthralgia and a sore throat arrived at our hospital by ambulance. She denied having diarrhea or abdominal pain, although she reported dysphagia, dysphonia and salivation. Levofloxacin (LVFX) (500 mg/day) had been prescribed just two days before she visited our hospital. At 20 years of age, she had been diagnosed with schizophrenia and prescribed sodium valproate (400 mg/day), risperidone ( $2 \mathrm{mg} / \mathrm{day})$, lorazepam $(1 \mathrm{mg} /$ day $)$ and estazolam $(2 \mathrm{mg} /$ day $)$.

Nine months before admission, she had been diagnosed with Grave's disease and prescribed methimazole (MMI). She had no history of smoking, alcohol intake or surgery, including splenectomy. She had no sick contact. Seven months earlier, her white blood cell count had been 6,100 cells $/ \mathrm{mm}^{3}$ (normal range: 3,900-9,800 cells $/ \mathrm{mm}^{3}$ ). However, this parameter decreased to 1,000 cells $/ \mathrm{mm}^{3}$ two days before

${ }^{1}$ Department of Respiratory Medicine and Internal Medicine, Akashi Medical Center, Japan and ${ }^{2}$ Department of Internal Medicine, Kakogawa West City Hospital, Japan

Received for publication January 24, 2013; Accepted for publication June 3, 2013

Correspondence to Dr. Naoto Ishimaru, maru-tkb@umin.ac.jp 
Table. Laboratory Findings on Admission

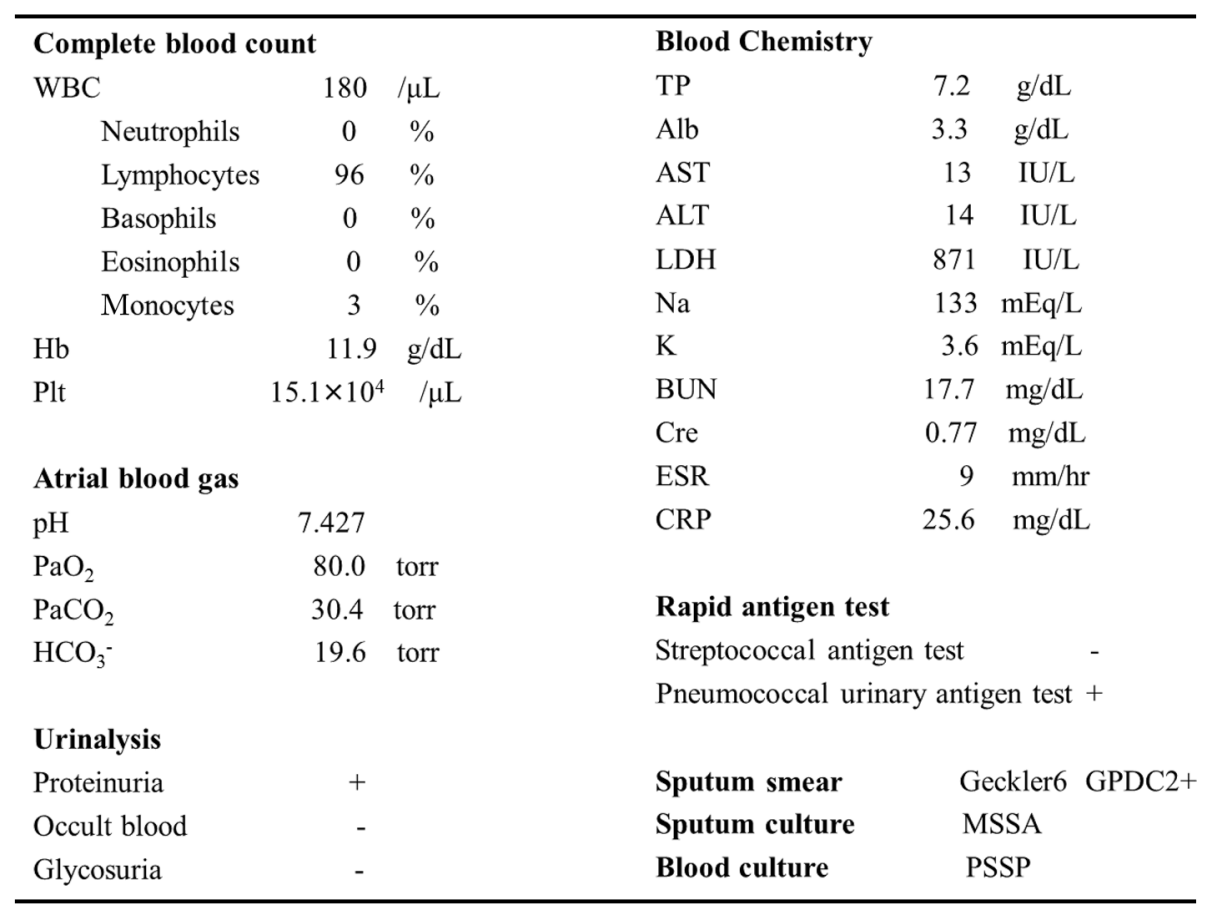

admission. Upon examination, the patient appeared sick and had difficulty breathing with drooling. Her weight was 68.8 $\mathrm{kg}$, her height was $161 \mathrm{~cm}$ and her body mass index was $26.5 \mathrm{~kg} / \mathrm{m}^{2}$. Her level of consciousness was E4V2M4 on the Glasgow Coma Scale. Her body temperature was $40.4^{\circ} \mathrm{C}$, her heart rate was $178 \mathrm{bpm}$, her blood pressure was 148/84 $\mathrm{mmHg}$, her respiratory rate was 38 breaths/min and her oxygen saturation was $96 \%$ while breathing ambient air. In addition, the tonsils were swollen with pus, and the cervical lymph nodes were enlarged bilaterally. Pan-inspiratory stridor was heard in the neck. A cardiac examination revealed normal S1 and S2 sounds. The patient's abdomen was normal, and there was no peripheral edema. The results of a neurologic examination were unremarkable.

The laboratory findings obtained on admission are shown in Table. The white blood cell count was markedly decreased at 180 cells $/ \mathrm{mm}^{3}$ (normal range: 3,900-9,800 cells/ $\mathrm{mm}^{3}$ ) with agranulocytosis. The lactate dehydrogenase level was elevated at $871 \mathrm{IU} / \mathrm{L}$ (normal range: 120-240 IU/L). The C-reactive protein level was markedly elevated at 25.6 $\mathrm{mg} / \mathrm{dL}$ (normal range: $<0.30 \mathrm{mg} / \mathrm{dL}$ ).

Immediately after arrival, we passed a laryngoscope down the patient's throat into the larynx. A laryngoscopic observation revealed that the upper airway mucosa was markedly swollen with pus from the adenoid to the larynx, with pinhole stenosis in the airway. The epiglottis and larynx were not significantly inflamed. Therefore, the patient was intubated.

Electrocardiography revealed sinus tachycardia with evidence of left-axis deviation. Chest radiography revealed no infiltration. Computed tomography of the neck and chest showed areas of soft tissue density fully wrapping the intra- tracheal tube from the pharynx to the larynx (Fig. 1). A sputum smear with Gram staining revealed Gram-positive diplococci. The results of the Binax Streptococcus Pneumoniae Urinary Antigen Kit (Binax, Portland, ME, USA) were positive. Following admission to the intensive care unit, the patient's systolic blood pressure decreased to $60 \mathrm{mmHg}$. Fluid resuscitation with acetated Ringer's solution was administered. Methimazole was discontinued. Sodium valproate, risperidone, lorazepam and estazolam were continued. In addition to $4 \mathrm{~g} /$ day of ceftriaxone (CTRX), $100 \mu \mathrm{g} / \mathrm{day}$ of granulocyte colony-stimulating factor (G-CSF; lenograstim) was administered. Thereafter, the patient's general condition, blood pressure and laboratory parameters of inflammation gradually improved in parallel with a recovery in the neutrophil count. The administration of G-CSF was continued for six days until the neutrophil count reached $2,000 \mu / \mathrm{L}$ on day 7. A sputum culture performed on admission yielded methicillin-susceptible Staphylococcus aureus. However, the organism cultured from two sets of blood cultures obtained on admission was confirmed to be penicillin-susceptible Streptococcus pneumoniae (PSSP). A seven-day course of CTRX was followed by $8 \mathrm{~g} /$ day of ampicillin for seven days. A blood culture performed on day 8 yielded no microbes. On day 9, the patient was successfully extubated, and the swelling in the upper airway soft tissue improved. On day 15 , she developed hyperthyroidism, and we administered $100 \mathrm{mg} /$ day of potassium iodide to control the thyroid function. The patient's clinical course is summarized in Fig. 2. 


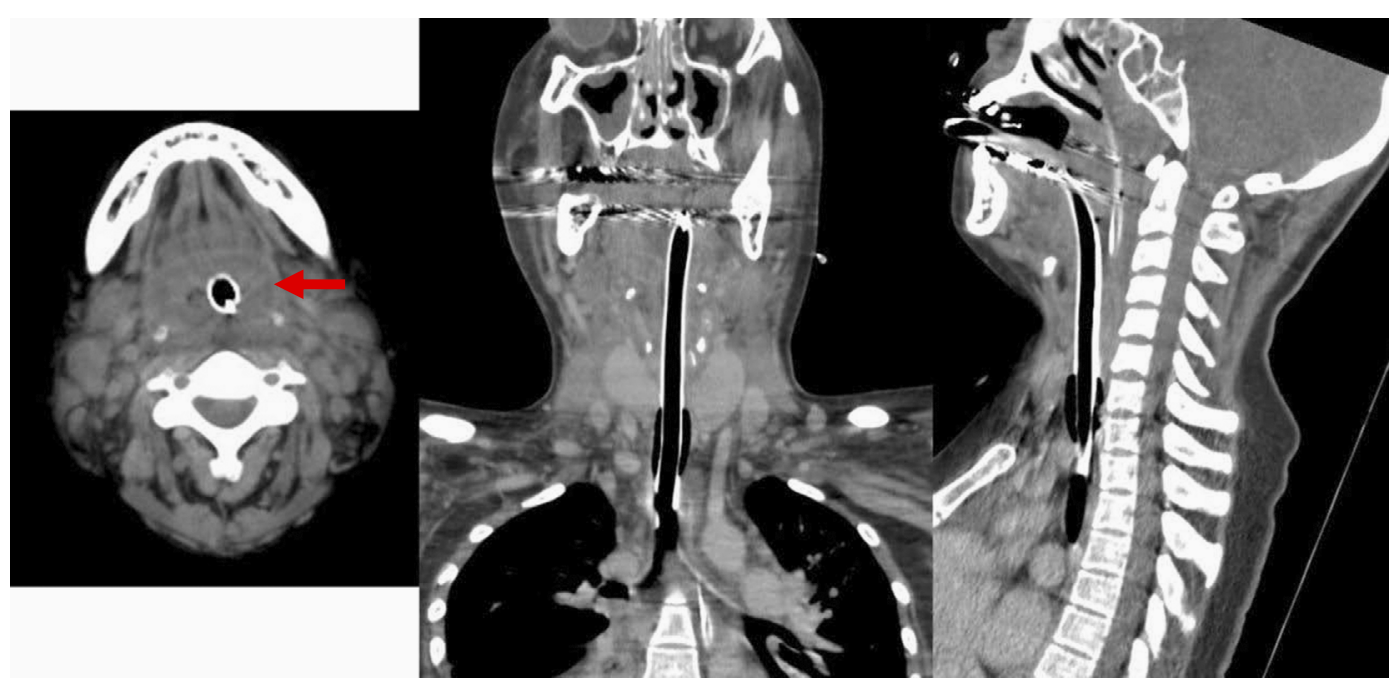

Figure 1. Computed tomography of the neck and upper chest revealing inflammatory change of soft tissues (arrow) adjacent to the intratracheal tube ranged from the pharynx to the larynx.

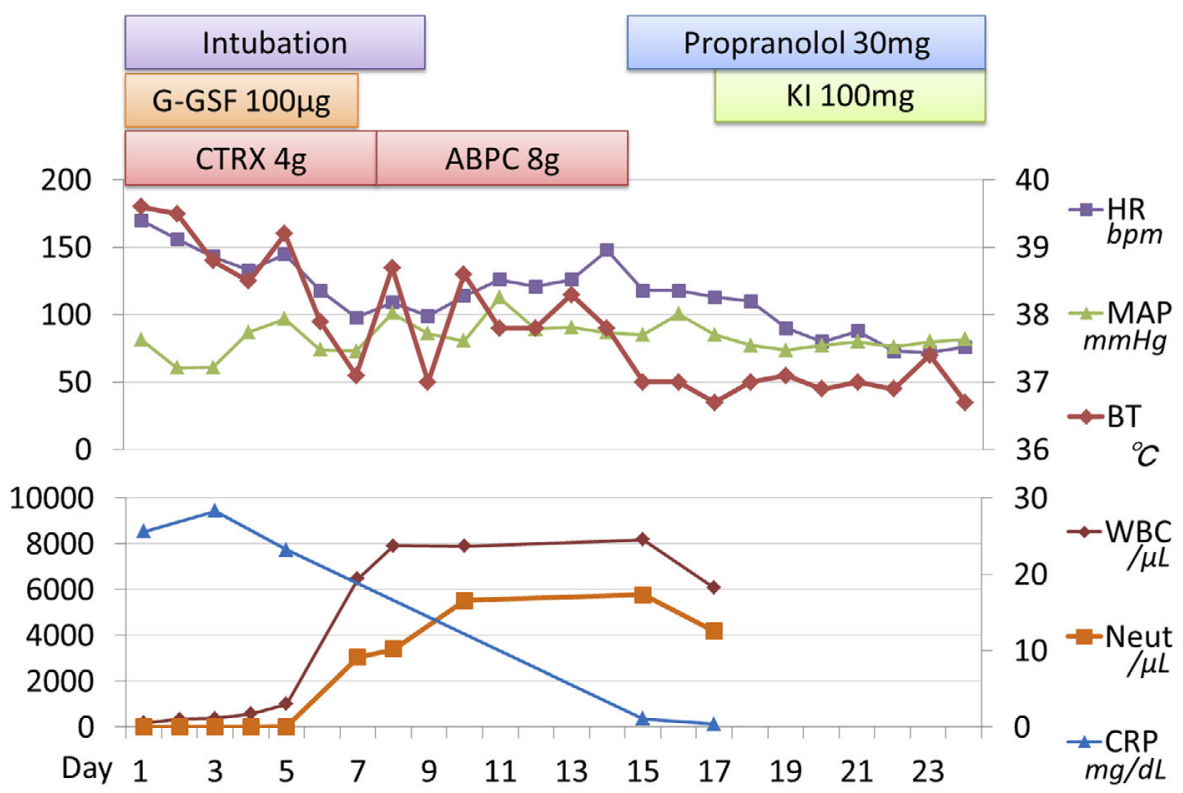

Figure 2. Clinical course of the patient. G-CSF: Granulocyte Colony-Stimulating Factor, CTRX: Ceftriaxone, ABPC: Ampicillin, KI: Potassium iodide

\section{Discussion}

In this case report, we described the case of a patient with ATD-induced agranulocytosis complicated by pneumococcal sepsis and upper airway obstruction. Antidepressants and antipsychotics were continued during the patient's clinical course of recovery from agranulocytosis, which argues against a causal relationship between these drugs and agranulocytosis in this case. Neutropenia itself can be caused by serious infections; however, the present patient had none of the following risk factors for sepsis: asplenia, hematologic malignancy, infectious diseases, such as HIV or influenza, or a history of medications, such as high-dose corticosteroids. In cases of neutropenia caused by Strepto- coccus pneumoniae infection, the patient's condition usually recovers rapidly following the administration of G-CSF (7). However, in this case, it took seven days for the patient to recover from neutropenia, even after receiving G-CSF, another finding that supports our contention that the agranulocytosis preceded the pneumococcal bacteremia. Agranulocytosis usually occurs within two months of the initiation of ATD therapy (8) and is more likely to develop in older patients ( $>40$ years of age) (9) and those receiving higher doses of MMI (30 vs. $15 \mathrm{mg} /$ day) (10). In the present young patient, agranulocytosis developed nine months after the administration of $30 \mathrm{mg} /$ day of MMI; therefore, we believe that the higher dose may have played a role in the pathogenesis of agranulocytosis in this case. Awareness of the possibility for the development of agranulocytosis re- 
mains important, even two months after the initiation of ATD-therapy.

Patients with ATD-induced agranulocytosis usually present with fever and a sore throat (11), with the most common clinical diagnoses being acute pharyngitis and tonsillitis (2). Severe pyogenic infections, such as peritonsillar and retropharyngeal abscesses, airway obstruction, pneumonia and acute respiratory distress syndrome may complicate ATD-induced agranulocytosis (2). The possibility for the sudden onset of life-threatening complications warrants the timely diagnosis and appropriate management of these infections. Despite the severe airway obstruction observed in the present case, we successfully treated the patient with emergency airway management and the administration of intravenous antibiotics and G-CSF.

A previous small randomized study suggested that G-CSF does not improve the hematologic recovery from ATDinduced agranulocytosis (12). Meanwhile, G-CSF has been reported to shorten the duration of neutropenia in patients with drug-induced agranulocytosis in other studies $(13,14)$ and to reduce mortality in a meta-analysis of 118 published reports (15). The present findings also support the effectiveness of G-CSF in treating ATD-induced agranulocytosis.

In the present case, PSSP was identified in blood culture bottles. Although a sputum smear with Gram staining revealed Gram-positive diplococci and the results of the $S$. pneumoniae Urinary Antigen Kit were positive, the sputum culture yielded no growth of $S$. pneumoniae. The specificity of sputum smears with Gram staining and the Urinary Antigen Kit is relatively high $(16,17)$, while the sensitivity of sputum cultures for $S$. pneumoniae bacteremia is reported to be $79 \%$ (18). The rate of culture positivity for S. pneumoniae decreases after antibiotic therapy (19). In the present case, the sputum sample was collected two days after the administration of LVFX. The delay until the assay of the sputum specimen may have resulted in the autolysis of the bacteria, which led to the negative culture result.

In the present case, the patient was initially treated with levofloxacin. The susceptibility of PSSP to levofloxacin was preserved (MIC $<1 \mu \mathrm{g} / \mathrm{mL}$ ) and found to be similar to that of most current PSSP strains (20). However, the treatment was ineffective under the state of agranulocytosis (5). Although the susceptibility of PSSP to ceftriaxone was preserved $(\mathrm{MIC}=0.5 \mu \mathrm{g} / \mathrm{mL}$ ), the treatment did not demonstrate any clinical effectiveness until the patient recovered from the agranulocytosis. The present case illustrates the significance of a patient's recovery from agranulocytosis when managing life-threatening infections.

In conclusion, the presence of $S$. pneumoniae sepsis must be considered in patients with ATD-induced agranulocytosis primarily involving the upper airway. In addition, emergency airway management and the administration of intravenous antibiotics and G-CSF should be provided in patients with ATD-induced agranulocytosis complicated by life-threatening infections.
The authors state that they have no Conflict of Interest (COI).

\section{References}

1. Cooper DS. Antithyroid drugs. N Engl J Med 311: 1353-1362, 1984.

2. Sheng WH, Hung CC, Chen YC, et al. Antithyroid-drug-induced agranulocytosis complicated by life-threatening infections. QJM 92: 455-461, 1999.

3. Andres E, Maloisel F. Idiosyncratic drug-induced agranulocytosis or acute neutropenia. Curr Opin Hematol 15: 15-21, 2008.

4. Julia A, Olona M, Bueno J, et al. Drug-induced agranulocytosis: prognostic factors in a series of 168 episodes. Br J Haematol 79: 366-371, 1991.

5. Kumashi P, Girgawy E, Tarrand JJ, Rolston KV, Raad II, Safdar A. Streptococcus pneumoniae bacteremia in patients with cancer: disease characteristics and outcomes in the era of escalating drug resistance (1998-2002). Medicine (Baltimore) 84: 303-312, 2005.

6. Tumbarello M, Tacconelli E, Caponera S, Cauda R, Ortona L. The impact of bacteraemia on HIV infection. Nine years experience in a large Italian university hospital. J Infect 31: 123-131, 1995.

7. Araya J, Katori M, Ishihara H, et al. Severe pneumococcal pneumonia with acute respiratory failure and neutropenia. Nihon Kokyuki Gakkai Zasshi 36: 803-808, 1998.

8. Tajiri J, Noguchi S, Murakami T, Murakami N. Antithyroid druginduced agranulocytosis. The usefulness of routine white blood cell count monitoring. Arch Intern Med 150: 621-624, 1990.

9. Cooper DS, Goldminz D, Levin AA, et al. Agranulocytosis associated with antithyroid drugs. Effects of patient age and drug dose. Ann Intern Med 98: 26-29, 1983.

10. Takata K, Kubota S, Fukata S, et al. Methimazole-induced agranulocytosis in patients with Graves' disease is more frequent with an initial dose of $30 \mathrm{mg}$ daily than with $15 \mathrm{mg}$ daily. Thyroid 19: 559-563, 2009.

11. Meyer-Gessner M, Benker G, Lederbogen S, Olbricht T, Reinwein D. Antithyroid drug-induced agranulocytosis: clinical experience with ten patients treated at one institution and review of the literature. J Endocrinol Invest 17: 29-36, 1994.

12. Fukata S, Kuma K, Sugawara M. Granulocyte colony-stimulating factor (G-CSF) does not improve recovery from antithyroid druginduced agranulocytosis: a prospective study. Thyroid 9: 29-31, 1999.

13. Andres E, Kurtz JE, Perrin AE, Dufour P, Schlienger JL, Maloisel F. Haematopoietic growth factor in antithyroid-drug-induced agranulocytosis. QJM 94: 423-428, 2001.

14. Sprikkelman A, de Wolf JT, Vellenga E. The application of hematopoietic growth factors in drug-induced agranulocytosis: a review of 70 cases. Leukemia 8: 2031-2036, 1994.

15. Beauchesne MF, Shalansky SJ. Nonchemotherapy drug-induced agranulocytosis: a review of 118 patients treated with colonystimulating factors. Pharmacotherapy 19: 299-305, 1999.

16. Anevlavis S, Petroglou N, Tzavaras A, et al. A prospective study of the diagnostic utility of sputum Gram stain in pneumonia. J Infect 59: 83-89, 2009.

17. Skoff TH, Farley MM, Petit $S$, et al. Increasing burden of invasive group B streptococcal disease in nonpregnant adults, 1990-2007. Clin Infect Dis 49: 85-92, 2009.

18. Garcia-Vazquez E, Marcos MA, Mensa J, et al. Assessment of the usefulness of sputum culture for diagnosis of community-acquired pneumonia using the PORT predictive scoring system. Arch Intern Med 164: 1807-1811, 2004.

19. Baggett HC, Rhodes J, Dejsirilert S, et al. Pneumococcal antigen testing of blood culture broth to enhance the detection of Streptococcus pneumoniae bacteremia. Eur J Clin Microbiol Infect Dis 31: 753-756, 2012. 
Intern Med 52: 2355-2359, 2013 DOI: 10.2169/internalmedicine.52.0220

20. Yamaguchi T, Hashikita G, Takahashi S, Itabashi A, Yamazaki T, Maesaki S. In vitro activity of beta-lactams, macrolides, telithromycin, and fluoroquinolones against clinical isolates of Strepto- coccus pneumoniae: correlation between drug resistance and genetic characteristics. J Infect Chemother 11: 262-264, 2005.

(C) 2013 The Japanese Society of Internal Medicine http://www.naika.or.jp/imonline/index.html 